\title{
The role of an immune checkpoint score in resected non-small cell lung cancer patients' prognosis
}

\author{
Rossana Berardi, Silvia Rinaldi \\ Clinica Oncologica, Università Politecnica Marche, Azienda Ospedaliero-Universitaria Ospedali Riuniti Umberto I, GM Lancisi, G Salesi di Ancona, Italy \\ Correspondence to: Prof. Rossana Berardi. Clinica Oncologica, Università Politecnica Marche, Azienda Ospedaliero-Universitaria Ospedali Riuniti \\ Umberto I, GM Lancis, G Salesi di Ancona, Via Conca 71-60126, Ancona, Italy. Email: r.berardi@univpm.it. \\ Provenance: This is an invited Editorial commissioned by Section Editor Dr. Chunlin Ou (Cancer Research Institute of Central South University, \\ Changsha, China). \\ Comment on: Usó M, Jantus-Lewintre E, Calabuig-Fariñas S, et al. Analysis of the prognostic role of an immune checkpoint score in resected non- \\ small cell lung cancer patients. Oncoimmunology 2016;6:e1260214.
}

Submitted Aug 15, 2017. Accepted for publication Sep 17, 2017.

doi: $10.21037 /$ jtd.2017.09.130

View this article at: http://dx.doi.org/10.21037/jtd.2017.09.130

Lung cancer is the leading cause of cancer related death in both sex and its incidence is still increasing. Non-small cell lung cancer (NSCLC) accounts for approximately $85 \%$ of all lung cancers (1). Despite many treatment options are available, most of NSCLC patients' diagnoses are reached in advanced stages and their prognosis remains poor. Over the past few years, a variety of prognostic and predictive factors have been investigated in patients with NSCLC, and several prognostic models have been proposed to predict the outcome of patients with NSCLC (2). Nevertheless, the prognosis for patient with NSCLC remains dismal, and novel approaches are required in order to optimize patient outcomes and guide treatment decisions. Currently, an improvement in the knowledge of tumor hallmarks has underlined the important role of the immune system in the pathogenesis and progression of lung cancer. In fact, on one hand it is involved in the removal of pathogens and in the prompt resolution of inflammation related to an increased risk of lung cancer occurrence; on the other hand, the immune system is able to detect and eliminate tumor cells, recognizing tumor-specific antigens or altered molecules induced by DNA damage (3). However, tumor cells can develop escape mechanisms, producing surface and soluble factors, involved in the modulation of immune-activation pathways. In particular, specific immune checkpoint factors, such as programmed death-1/programmed death-ligand 1 (PD-1/PD-L1), have been investigated in lung cancer, showing an important role in the immune cells suppressions and tumor escape. Specific PD-1 or PD-L1 immune checkpoint inhibitor agents have been developed, showing a relevant efficacy in advanced NSCLC patients $(4,5)$ and PD-L1 expression, in tumor cells and in tumor microenvironment, seems also to correlate with tumor response to some immune checkpoint inhibitors (6). However, the prognostic and predictive role of PD-L1 expression is still controversial, because patients with negative expression can respond to immunotherapy, as well as among patients with high tumor PD-L1 levels there are "not-responders" with poor prognosis (7). Nowadays, several on-going trials are evaluating the role of immunotherapy in early NSCLC stages and emerging data are showing that the immune system and immunecheckpoint inhibitors have an important role also in this setting. A recent retrospective study analysed lung adenocarcinoma tissues and their microenvironment, showing that these neoplastic lesions were enriched in TREG (regulatory $T$ cells), non-functional $T$ cells and have a strongly reduced CD8+ $\mathrm{T}$ effector, compared to normal lung tissue. Furthermore, the distinct tumorinfiltrating lymphocytes (TIL) signature was characterised by significant numerical and functional reduction of tumor CD16+ natural killer (NK) cells. In addition, clusters of PD-L1+ macrophages at the tumor-invasive margins were identified, suggesting their potential contribution in tumor $\mathrm{T}$ cells entry and modulation and so a potential role of immunotherapy also in earlier stages NSCLC (8). On 
the basis of these evidences, several studies evaluated the expression of immune-checkpoints molecules in tumor-cells and in TIL, in order to understand their prognostic and predictive role. Cytotoxic T-lymphocyte-associated protein 4 (CTLA-4) seems to be correlated to good prognosis whether expressed in primary tumor-cells but worse prognosis when expressed in metastatic lymph nodes (9). PD-L1 expression seems to correlate to worse prognosis in early stages NSCLC, with higher risk of lymph nodes metastases, higher percentage of TILs and higher risk of tumor relapse $(10,11)$. Furthermore, PD-L1 expression seems not to be correlated to a survival improvement in patients with resected NSCLC, receiving adjuvant chemotherapy (12). These data suggest that immune checkpoint activation has an important prognostic role in early stages NSCLC, however the detection of a single immune-factor expression is not enough to define patient prognosis and tumor microenvironment with several other activated pathways could have a crucial role in the tumor immune response modulation and in patients' outcome.

In a recent issue of the Oncoimmunology, Usó et al. published a retrospective, single center analysis of the expression of 20 immune-related genes, in primary lung tumor and in adjacent noncancerous lung tissues of 178 patients with resected NSCLC, using RTqPCR. According to gene expression, patients were divided in two groups, cluster I with lower expression of analyzed genes and cluster II with higher genes expression levels, showing that patients in cluster II had a better prognosis with longer relapse free survival (RFS) and overall survival (OS). Evaluating individual immune-related genes, the higher expression of IL23A, LGALS, CTLA4 and IL10 in tumor tissue was associated with a better outcome. In order to provide a more accurate prediction than a model using single genes, the authors ordered genes according to their prognostic power and created an immune checkpoint score (ICS) based on the expression of two genes, PD-1 and CTLA4, associated with longer survival. Patients with high ICS had longer RFS (82.6 vs. 23 months, $\mathrm{P}=0.009$; Figure 3) and OS (NR vs. 40.4 months, $\mathrm{P}=0.008$ ), and the prognostic role of ICS was confirmed at the multivariate analysis including also analytical and clinic-phatological variables such as lymph node involvement, KRAS status, cluster classification, individual immune gene expression $(\mathrm{P}=0.028)$ (13). These evidences are confirmed by a recent retrospective study that demonstrated the prognostic role of an immune signature of 25 genes, clustering patients in 3 different risk-groups and underlying that patients of high risk group presented higher neutrophil infiltration and necrosis in tumor tissues (14). Although this is a pioneering retrospective study and results should be confirmed by prospective studies, this study reinforces the idea that NSCLC patients with the activation of immune system and highest expression levels of immunerelated genes had better outcomes and that ICS is a prognostic factor to take into account in the management of early-stage NSCLC patients.

In conclusion, immune system plays a crucial role in carcinogenesis and prognosis of early-stages NSCLC and immunotherapy represents an effective treatment option. The detection of ICS is important to assess early-stage NSCLC patients' outcome and it might represent a valid item for diagnosis and therapeutic strategy of early-stages NSCLC. Furthermore, ICS based on the expression of two genes, $P D-1$ and CTLA4, could be useful to detect the best therapeutic approach also for end-stage NSCLC patients. In particular, it could help to determine which patients could benefit from a single immune agent, such as anti PD-1 or anti PD-L1, or those who benefit more from the combination of immune checkpoint inhibitors, such as anti CTLA-4 and anti-PD-1. In the future, the predictive role of ICS should be investigated and this approach could be integrated with other clinic-phatological variables for a better understanding of the pathogenic mechanisms underlying lung cancer and therefore used to improve patient outcomes through tailored therapy.

\section{Acknowledgements}

None.

\section{Footnote}

Conflicts of Interest: The authors have no conflicts of interest to declare.

\section{References}

1. Jemal A, Bray F, Center MM, et al. Global cancer statistics. CA Cancer J Clin 2011;61:69-90.

2. Berardi R, Rinaldi S, Santoni M, et al. Prognostic models to predict survival in patients with advanced non-small cell lung cancer treated with first-line chemo- or targeted therapy. Oncotarget 2016;7:26916-24.

3. Dunn GP, Bruce AT, Ikeda H, et al. Cancer immunoediting: from immunosurveillance to tumor escape. Nat Immunol 2002;3:991-8. 
4. Borghaei H, Paz-Ares L, Horn L, et al. Nivolumab versus docetaxel in advanced nonsquamous non-small-cell lung cancer. N Engl J Med 2015;373:1627-39.

5. Herbst RS, Baas P, Kim DW, et al. Pembrolizumab versus docetaxel for previously treated, $\mathrm{PD}-\mathrm{L} 1$-positive, advanced non-small-cell lung cancer (KEYNOTE-010): a randomised controlled trial. Lancet 2016;387:1540-50.

6. Peters S, Gettinger S, Johnson ML, et al. Phase II trial of atezolizumab as first-line or subsequent therapy for patients with programmed death-ligand 1-selected advanced non-small-cell lung cancer (BIRCH). J Clin Oncol 2017;35:2781-9.

7. Gridelli C, Ardizzoni A, Barberis M, et al. Predictive biomarkers of immunotherapy for non-small cell lung cancer: results from an Experts Panel Meeting of the Italian Association of Thoracic Oncology. Transl Lung Cancer Res 2017;6:373-86.

8. Lavin Y, Kobayashi S, Leader A, et al. Innate immune landscape in early lung adenocarcinoma by paired singlecell analyses. Cell 2017;169:750-65.e17.

9. Paulsen EE, Kilvaer TK, Rakaee M, et al. CTLA-4 expression in the non-small cell lung cancer patient tumor microenvironment: diverging prognostic impact in primary tumors and lymph node metastases. Cancer Immunol Immunother 2017. [Epub ahead of print].

10. Zhang M, Wang D, Sun Q, et al. Prognostic significance of PD-L1 expression and 18F-FDG PET/CT in surgical pulmonary squamous cell carcinoma. Oncotarget 2017;8:51630-40.

11. He Y, Rozeboom L, Rivard CJ, et al. PD-1, PD-L1 protein expression in non-small cell lung cancer and their relationship with tumor-infiltrating lymphocytes. Med Sci Monit 2017;23:1208-16.

12. Tsao MS, Le Teuff G, Shepherd FA, et al. PD-L1 protein expression assessed by immunohistochemistry is neither prognostic nor predictive of benefit from adjuvant chemotherapy in resected non-small cell lung cancer. Ann Oncol 2017;28:882-9.

13. Usó M, Jantus-Lewintre E, Calabuig S, et al. Analysis of the prognostic role of an immune checkpoint score in resected non-small cell lung cancer patients. Oncoimmunology 2016;6:e1260214.

14. Li B, Cui Y, Diehn M, et al. Development and validation of an individualized immune prognostic signature in earlystage nonsquamous non-small cell lung cancer. JAMA Oncol 2017. [Epub ahead of print].
Cite this article as: Berardi $\mathrm{R}$, Rinaldi $\mathrm{S}$. The role of an immune checkpoint score in resected non-small cell lung cancer patients' prognosis. J Thorac Dis 2017;9(10):3480-3482. doi:10.21037/jtd.2017.09.130 